scription and pre-mRNA splicing at their surface. Methods: To decipher whether PIP2containing NLIs surface recruit a regulatory proteins abovementioned processes, we employed a comparative quantitative mass spectrometry (MS) analysis of PIP2 nuclear fraction combined with super-resolution microscopy visualization of candidate proteins. Results: Our quantitative MS approach identified more than 300 putative NLIs proteins, which represents $35 \%$ of nuclear PIP2 - associated proteins. Moreover, $50 \%$ of those proteins are connected to gene expression and more that 30 $\%$ to RNA processing. Super-resolution microscopy showed that candidate proteins form foci in nucleoplasm and associate with sub-population of NLIs. In contrary, proteins predicted by MS as NLIs-nonassociated showed random distribution in respect to nucleoplasmic PIP2 foci. Conclusions: It is known that RNA splicing occurs co-transcriptionally. We show that sub-population of NLIs interact with regulators of both RNAPII transcription and pre-mRNA splicing [2]. We hypothesis that the surface of NLIs facilitate spatiotemporal regulation of these processes.

This study was supported by the Czech Academy of Sciences (JSPS-18-18); Grant Agency of the Czech Republic (16-03346S, 17-09103S, 15-08738S); Technology Agency of the Czech Republic (TE01020118); IMG ASCR, v. v. i.(RVO: 68378050); European Regional Development Fund (CZ.02.1.01/0.0/0.0/16 013/0001775 ). Microscopy Centre - IMG AS CR the Czech-Biolmaging project (LM2015062 funded by MEYS CR).

References: 1. Sobol, M., et al., Nuclear phosphatidylinositol 4,5-bisphosphate islets contribute to efficient RNA polymerase II-dependent transcription. J Cell Sci, 2018. 131(8). 2. Sztacho, M., et al., Nuclear phosphoinositides and phase separation: Important players in nuclear compartmentalization. Adv Biol Regul, 2018. doi: http://dx.doi.org/10.7124/bc.0009B4

\section{Nuclear Vinculin involvement in mouse spermatogenesis}

Petr Flachs $^{1}$, Alzbeta Darasova ${ }^{1}$, Pavel Hozak ${ }^{1,2}$

${ }^{1}$ Laboratory of Epigenetics of the Cell Nucleus, Institute of Molecular Genetics of the ASCR, division BIOCEV, Prague, Czech Republic; ${ }^{2}$ Laboratory of Biology of the Cell Nucleus, Institute of Molecular Genetics of the ASCR, Prague, Czech Republic petr.flachs@img.cas.cz

The focal adhesion protein vinculin (VCL) is a cytoskeletal protein associated with cell-matrix junctions where it acts as a molecular clutch between the actin cytoskeleton and the extracellular matrix via talins and integrins. However, our study reveals new localization and unexpected role of VCL in the nuclei of spermatocytes during Prophase I. In particular, VCL localizes in the nuclear interior along the meiosisspecific structure synaptonemal complex (SC) and in the centromeric regions of homologous chromosomes. To understand the role of VCL in the early meiotic division, we prepared a VCL conditional knock-out mouse model (VCL cKO) where the VCL deletion is manifested explicitly during the early stages of spermatogenesis. In comparison with the wild-type control, our mouse model shows significantly decreased ability of males to sire offspring together with increased percentage of malformed sperm. According to our short-term cultivation experiments primary spermatocytes of VCL cKO males exhibit deficiency in progression throughout late prophase I. Likewise, FACS (fluorescence-activated cell sorting) analysis revealed the enrichment of the prophase I spermatocytes. The chromosomal pairing and the crossing-over formation appear intact. However, spermato- 
cytes in late prophase I show a premature separation of homologous chromosomes with partial loss of the central element of SC and significantly increased distance between the centromeres. Taken together, our findings indicate that VCL plays an important role in the meiotic progression. We show that the VCL deletion in spermatocytes leads to considerable abnormalities on the level of chromosomal desynapsis which in the end results in the decreased breeding capability of VCL cKO males.

This study is supported by the CSMS scholarship, GACR (16-03403S), the project,BIOCEV“(CZ.1.05/1.1.00/02.0109) and Light Microscopy Core Facility, IMG ASCR.

doi: http://dx.doi.org/10.7124/bc.0009B5

\section{High-resolution study of epigenetic processes: new insights into methylation and demethylation}

\author{
S. Siciliani, I. Masiello, L. Zannino, F. Ba- \\ siricò, C. Casali, E. King, A. Lacavalla, \\ L. Saia, M. Scaltritti, M. Biggiogera \\ Laboratory of Cell Biology and Neurobiology, De- \\ partment of Biology and Biotechnology, University \\ of Pavia, Italy \\ stella.siciliani93@gmail.com
}

Methylation and demethylation are two epigenetic processes of a big relevance for different biological pathways. The two events happen on the carbon in position five of the cytosine belonging to the so called $\mathrm{CpG}$ island. The methylation implies the addition of a methyl group on the cytosine, forming the 5-methylcytosine $(5 \mathrm{mC})$ thanks to enzymes called DNMT (DnaMethyltransferase). After, when required, the methyl group is oxidized or demethylated by a family of enzyme called TET, forming the 5-hydroxymethylcytosine $(5 \mathrm{hmC})$. The role of the $5 \mathrm{mC}$ is generally correlated with gene expression repression, while the $5 \mathrm{hmC}$ function must be clarified. In this context, in order to elucidate the hypothetic role of these markers we decide to investigate at ultrastructural level, by looking at the distribution of two epigenetic modifications putting our attention on different areas of the cell nucleus. Our study where carried out by using transmission electron microscope, light microscope and molecular biology techniques. We observed that in condensed regions of the nucleus the DNA is always highly methylated rather than hydroxymethylated, but in the so called perichromatin region the pattern changes. Indeed, in this region it was possible to notice an abundancy of demethylation underlined both by the presence of the $5 \mathrm{hmC}$ and of the enzymes involved in the processes: TET2. This result could allow to hypothesize a sort of activating role for the oxidized modification respect to its reduced form and underline how the perichromatin region is a dynamic region where DNA status changes.

doi: http://dx.doi.org/10.7124/bc.0009B6

\section{Lamin A and PI(4,5)P2 - a novel complex in the cell nucleus}

Sara Escudeiro-Lopes, Vlada V. Philimonenko, Lenka Jarolimová, and Pavel Hozák

Department of Biology of the Cell Nucleus, Institute of Molecular Genetics ASCR v.v.i. Vídeňská 1083, 142 20, Prague 4, Czech Republic saraescudeiro.l@gmail.com

Lamins are important regulators of nuclear structural integrity as well as nuclear functional processes such as DNA transcription, replication and 